\title{
Preparations of DNA-aligned thin films by cast and LB methods and their anisotropic electric conductivity along DNA strands in the film
}

\author{
By Yoshio OKaHATA ${ }^{\dagger)}$ and Hajime NaKaYAMA \\ Department of Biomolecular Engineering, Tokyo Institute of Technology, \\ 4259, Nagatsuda, Midori-ku, Yokohama, Kanagawa 226-8501 \\ (Communicated by Seizo OKamuRa, M. J. A., Nov. 13, 2000)
}

\begin{abstract}
Organic thin films in which DNA double strands aligned in one direction were prepared by two methods: i) the polyion complex of DNA anions and cationic amphiphiles was prepared, the organic solution of the DNA-lipid complex was cast on the plate, and the obtained transparent film was stretched in one direction, and ii) DNA anions were solubilized in subphase with intercalated dyes and were transferred with cationic lipid monolayers by vertical dipping method. DNA strands could be aligned along stretching direction in the cast film and along dipping direction in the Langmuir-Blodgett (LB) film. The orientation of DNA strands in these thin films was confirmed by X-ray diffraction and polarized absorption spectra. The DNA-aligned films prepared by both cast-stretching and LB methods showed a large anisotropic and ohmic electric current $\left(10^{-3}-10^{-5} \mathrm{~S} \mathrm{~cm}^{-1}\right.$ ratio $\left.=10^{4}\right)$ along DNA strands in the film.
\end{abstract}

Key words: DNA; DNA-lipid complex; cast film; LB film; X-ray diffraction; polarized absorption spectra; anisotropic electric conductivity.

Introduction. Although DNA is welknown as a source of biological information depending on base sequences, it is also interesting as a molecular material that shows a rod-like, stereo-chemical double helical structure with $\pi$-electron-rich base-pair stacking: the base separation is $3.4 \AA$ while the diameter of the helix is about $20 \AA .^{1)}$ DNAs are soluble only in aqueous solutions and their fibrous crystals can be prepared by slow evaporation from the aqueous solution. Duplex structures in the fibers have been studied by X-ray diffrac$\operatorname{tion}^{2), 3)}$ and solid state NMR. ${ }^{4)-6)}$ Orientation of DNA strands by using hydrodynamic flow gradients in the dilute aqueous solution ${ }^{7,8)}$ and lyotropic liquid crystal properties of rod-like DNA in the concentrated aqueous solution ${ }^{9)}$ have been investigated. If a self-standing film in which rod-like DNA strands are aligned in one direction could be prepared by a simple method, it is interesting as molecular materials for electron transfer filed.

In this paper, we describe two methods to prepare organic thin films in which DNA strands aligned in one

†) Correspondence to: Y. Okahata. direction by i) uniaxial stretching of a cast film of DNAlipid complex and ii) vertical dipping Langmuir Blodgett (LB) method of anionic DNA strands with cationic lipid monolayers. The film structure was confirmed by X-ray diffraction and polarized absorption spectra of intercalated dye molecules. The thin films, in which DNA strands are aligned in one direction, could be expected as an electric conducting material, and the DNA-aligned films prepared by both methods showed a large anisotropic and ohmic electric current along stacked base-pairs of DNA strands aligned in the film.

Experimental section. Preparation of a cast film of a DNA-lipid Complex. An aqueous solution (100 $\mathrm{mL})$ of $\mathrm{DNA}^{-} \mathrm{Na}^{+}$from salmon testes $(0.50 \mathrm{~g}, \mathrm{Mw} c a$. $20,000,000, c a .30,000 \mathrm{bps})$ and an aqueous solution (100 $\mathrm{mL})$ of 1.1 eq. mol of cationic amphiphiles $\left(\mathrm{C}_{10}-4 \mathrm{G}-\mathrm{N}^{+}\right.$, $1.5 \mathrm{mmol}$ ) were mixed at room temperature and polyion-complex precipitates were gathered by centrifugation and freeze-dried. The white powder was solubilized in chloroform and reprecipitated to diethyl ether two times. The obtained DNA-lipid complex was confirmed by elemental analysis to form 1:1 complexes of 
a phosphate anion and the cationic amphiphile. The DNA-lipid complex was soluble only in organic solvents such as chloroform, benzene, and ethanol, but not in aqueous buffer solution.

The $\mathrm{CHCl}_{3} / \mathrm{EtOH}$ (4:1) solution $(10 \mathrm{~mL})$ of the DNA-lipid complex $(0.25 \mathrm{~g})$ was cast on a Teflon plate and the solvent was evaporated slowly under the saturated vapor at room temperature. The obtained selfstanding film was transparent ( $c a .60 \mathrm{\mu m}$ thick), waterinsoluble, and physically stable. The film could be

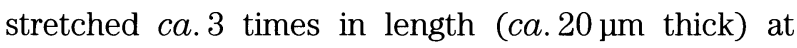
room temperature, which was still transparent and physically strong.

Preparation of a LB film of a DNA-lipid complex. Ten $\mathrm{mM} \mathrm{bp}^{-1}$ of DNA and then the respective concentration of intercalating dye molecules were solved in the subphase of Mili QII water. A chloroform solution of cationic dialkyl amphiphiles $\left(2 \mathrm{C}_{18}\right.$-glu- $\left.\mathrm{N}^{+}\right)$was spread on the subphase. After $1 \mathrm{hr}$, a monolayer was compressed and measured $\pi$-A isotherms. Monolayers were transferred on the hydrophobic quartz plate surface treated with 1,1,1,3,3,3-hexamethyldisilazane by using a conventional vertical dipping and a horizontal lifting method.

Transferred process of the DNA-lipid complex was followed by both the conventional barrier movement on the trough and the quartz-crystal microbalance (QCM) method. QCMs are known to provide very sensitive mass measuring devices because their vibration frequency decreases upon the deposition of mass on the $\mathrm{Au}$ electrode in nanogram level. ${ }^{10)-16)}$ We already reported that the transferred mass of LB films can be directly followed from the frequency decrease (mass increase) on the QCM substrate. ${ }^{11,12)}$ Calibration of the QCM used in this experiment gave the following equation according to Sauerbrey's equation. ${ }^{10)}$

$$
\Delta m=-(1.15 \pm 0.01) \times 10^{-9} \Delta F .
$$

The Au electrodes of the QCM were hydrophobized by treating with self-assembled monolayer of dodecanethiol, in order to transfer of LB films.

Measurements. The X-ray diffraction pattern of the DNA film was recorded on an imaging plate by using model R-AXIS, Rigaku-Denki, Co., Tokyo, Japan. The film was cut and piled up into a fine strip with a size of $8 \times 3$ mm for X-ray measurements.

Polarized absorption spectra of the DNA film intercalated with dyes were measured on a quartz plate by a model HP8452A diode array, Hewlett-Packard

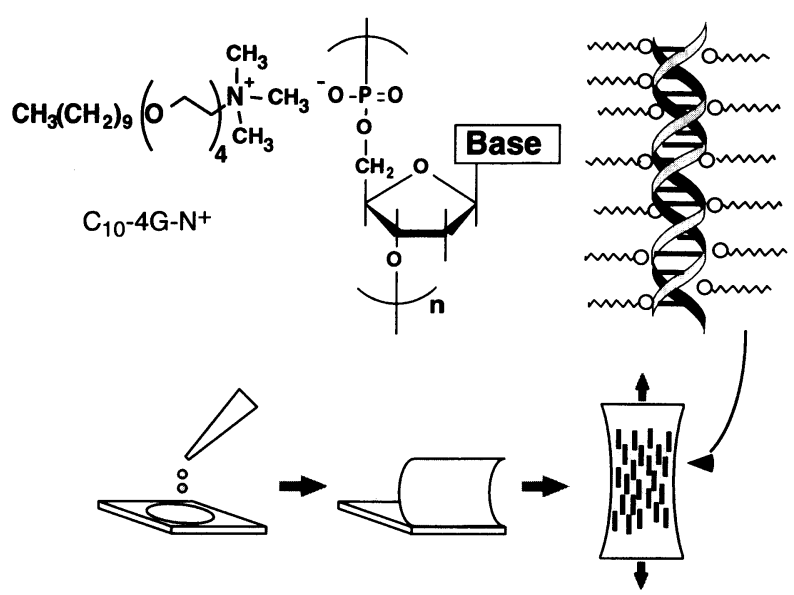

Fig. 1. A schematic illustration of preparation of a DNA-lipid complex cast film.

Japan Co., Ltd., Tokyo, Japan. The dye-intercalated film was prepared by soaking the film into aqueous solution $(25 \mathrm{mM})$ of ethidium bromide for a day at room temperature.

Electro conductivity was measured by using a comb-shaped Au electrode and a dc ammeter.

Results and discussion. Preparation of cast and stretched film of the DNA-lipid complex. Preparation step of cast film of DNA-lipid complex is shown in Fig. 1. The obtained self-standing film was transparent (ca.60 $\mathrm{mm}$ thick), water-insoluble, and physically stable. Fig. 2a shows X-ray diffraction patterns of an as-cast film of the DNA-lipid complex. When the incident beam was irradiated perpendicular to the film plane, the circular reflection with $41 \AA$ was observed that corresponds to a diameter of a DNA-lipid complex. When the beam was exposed parallel to the film plane (side-edge view), hexagonal spots with $41 \AA$ spacing were observed. They indicate the DNA-lipid strands whose diameter is $41 \AA$ A aggregate into hexagonal closepacked structures in the film plane. Thus, aggregates of hexagonal-packed DNA-lipid strands aligned on the film plane in random directions, as schematically shown in Fig. 2a.

The DNA-lipid cast film was stretched $c a .3$ times in length (ca. $20 \mu \mathrm{m}$ thick) in wet state, and X-ray diffraction patterns are shown in Fig. $2 \mathrm{~b}$. When the incident beam was irradiated parallel to the top edge of the stretched film, the circular reflection with $41 \AA$ was observed. When the beam was exposed parallel to the side edge and perpendicular to the film plane, diffraction on the equator appeared as two spots of $41 \AA$ indicating distance between DNA-lipid strands and the diffraction 
(a) As-cast Film

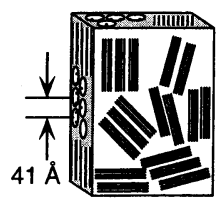

(b) Stretched Film in moisuture

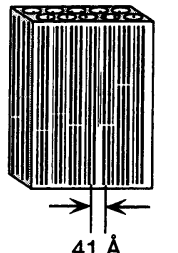

(c) Stretched Film in dry state

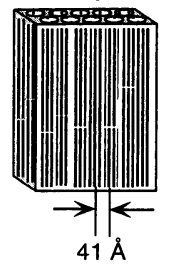

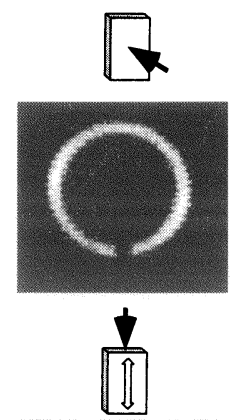
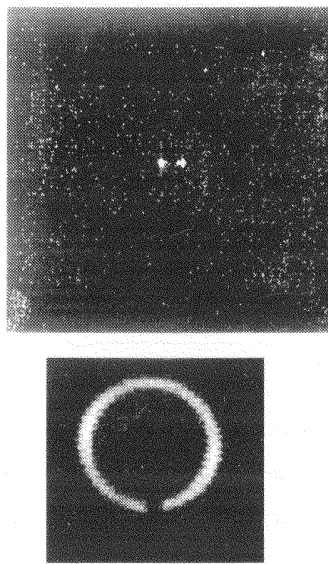
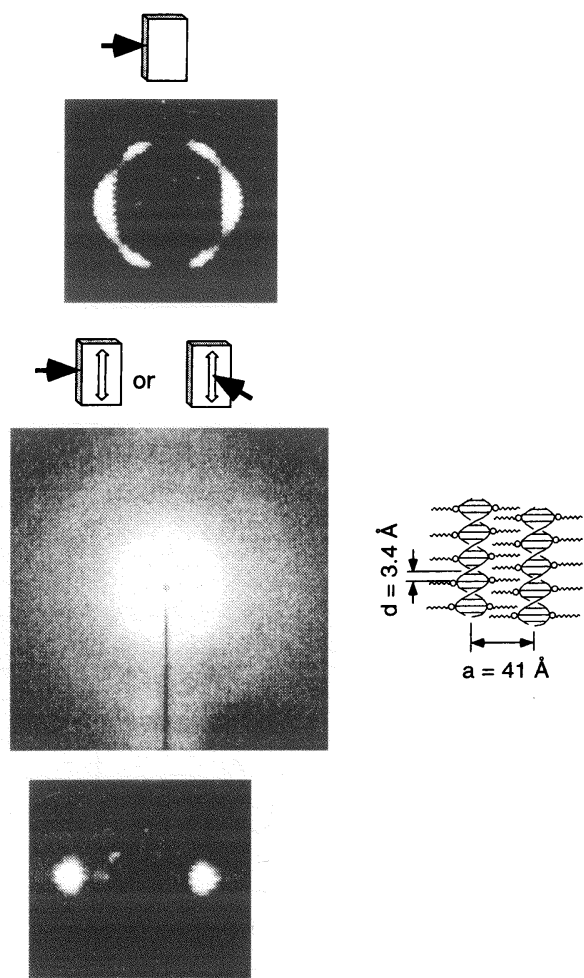

Fig. 2. X-ray diffraction patterns of (a) the as-cast film, (b) the stretched film of the DNA-lipid complex in water moisture, and (c) the stretched DNA-lipid film in dry state. Open, two-headed arrows show the stretching direction of the film. Closed arrow shows the incident beam of X-ray. X-ray photographs of (a) and (c) were enlarged to clearly see diffraction.

on the meridian of $3.4 \AA$ indicating distance between parallelly stacked base-pairs was clearly observed. ${ }^{2-4)}$ These findings clearly show that DNA strands aligned parallel to the stretched direction in the film, and basepairs stacked perpendicular to the direction of DNA strands as shown in the illustration. Neither the diameter nor the distance between strands ( $41 \AA$ ) was affected by stretching. When the stretched film was dried in air, only diffraction on the equator as two spots of $41 \AA$ were observed but not diffraction on the meridian for basepairs (Fig. 2c). This suggests that the orientation of base pairs is not perpendicular to the stretched direction, although DNA strands are aligned parallel to the stretched direction.

When the stretched DNA-lipid film was soaked in an aqueous solution of ethidium bromide $\left(\lambda_{\max }=480 \mathrm{~nm}\right)$ for a day at room temperature, the transparent film turned red $\left(\lambda_{\max }=520 \mathrm{~nm}\right)$ and the aqueous solution became clear. Thus, the ethidium intercalated completely between base pairs of the DNA film. When the film was moved into the new aqueous buffer solution, the intercalated dye molecules were hardly removed from the film at least for a day. Similar intercalation behavior into the film was observed for other dyes such as proflavine, acridine orange, and safranin T. ${ }^{17), 18)}$ Fig. 3a shows polarized absorption spectra of ethidium intercalated in the wet and stretched DNA-lipid film. The absorption at $520 \mathrm{~nm}$ of the light polarized perpendicularly to the stretched direction of the film was 3.3 times larger than that the light polarized parallel to the stretched direction. In the case of the as-cast DNA-lipid film, there were no differences in absorption of the parallel and perpendicular light. The large dichroic ratio of $\mathrm{A}_{\perp} / \mathrm{A}_{/ /}=3.3$ indicates that the intercalated dyes are aligned perpendicular to the stretched direction of the film: the DNA strands are aligned parallel to the stretched direction. The secondary order parameter (orientation moment) of DNA strands in the film was calculated to be $c a$. 0.7 from the $A_{\perp} / A_{/ /}=3.3$. This value is close to the orientation of synthetic rod-like polymers such as poly(arylenes) prepared by a uniaxicial stretching method. ${ }^{19), 20)}$ 

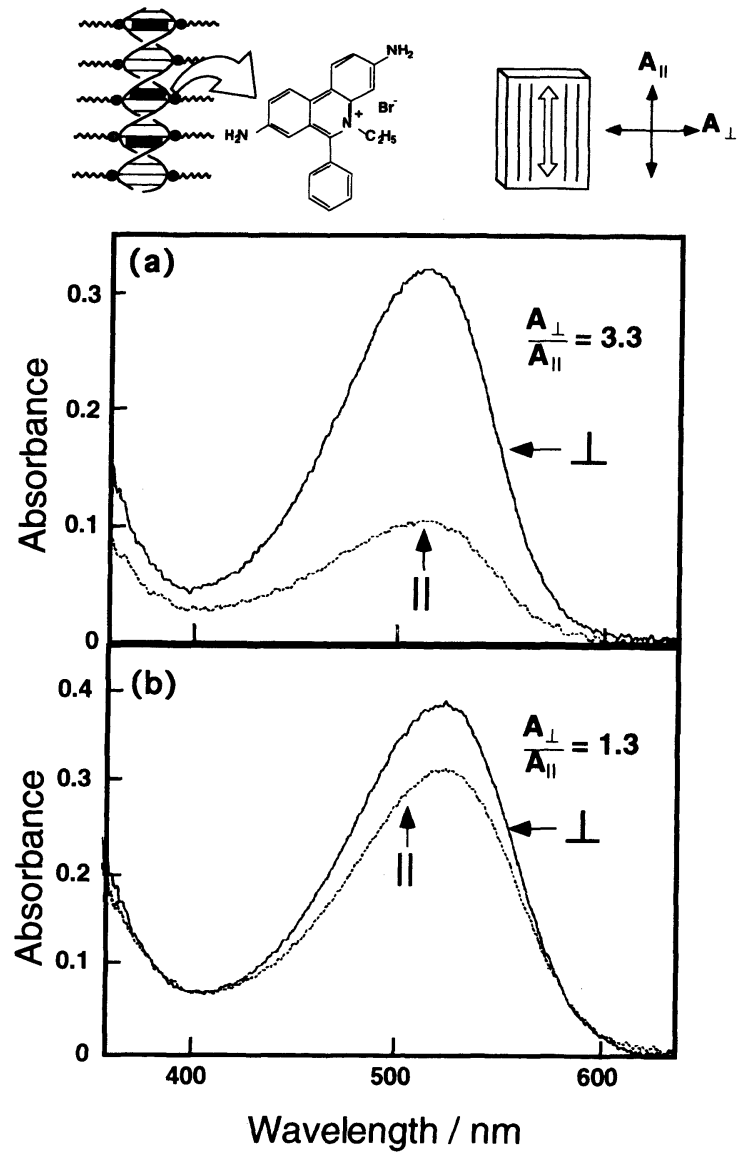

Fig. 3. Polarized absorption spectra of ethidium bromide intercalated into the stretched DNA-lipid film (a) in wet and (b) in the dry state. Ethidium bromide was intercalated one molecule per 10 base-pairs at $25^{\circ} \mathrm{C}$. Open, two-headed arrows show the stretching direction of the film.

When a polarized spectrum of the film was taken in the dry state, a small dichroic ratio was observed $\left(\mathrm{A}_{\perp} / \mathrm{A}_{/ /}\right.$ $=1.3$, see Fig. $3 \mathrm{~b}$ ). This is due to the slant structures of base pairs and intercalated dyes to the axis of strands, since DNA strands were confirmed to be aligned along the stretching direction even in the dry film (see Fig. 2c). These changes of polarized absorption spectra depending on water moisture were reversible at least 10 times.

Orientation of DNA strands during LB process. Rod-like polymers such as polyglutamate, ${ }^{21)}$ polysiloxane ${ }^{22)}$ alkylated cellulose, ${ }^{23)}$ and discotic crystals, ${ }^{24)}$ have been reported to form LB films in which rod-like molecules aligned in one direction during the compression process on the subphase ${ }^{25)}$ or the deposition process of monolayers. ${ }^{26)}$ DNA forms a rod-like strand and is a good candidate to form an oriented LB film in the same manner. ${ }^{27)}$ However, DNA is soluble only in water phase due to the anionic phosphate backbone. We

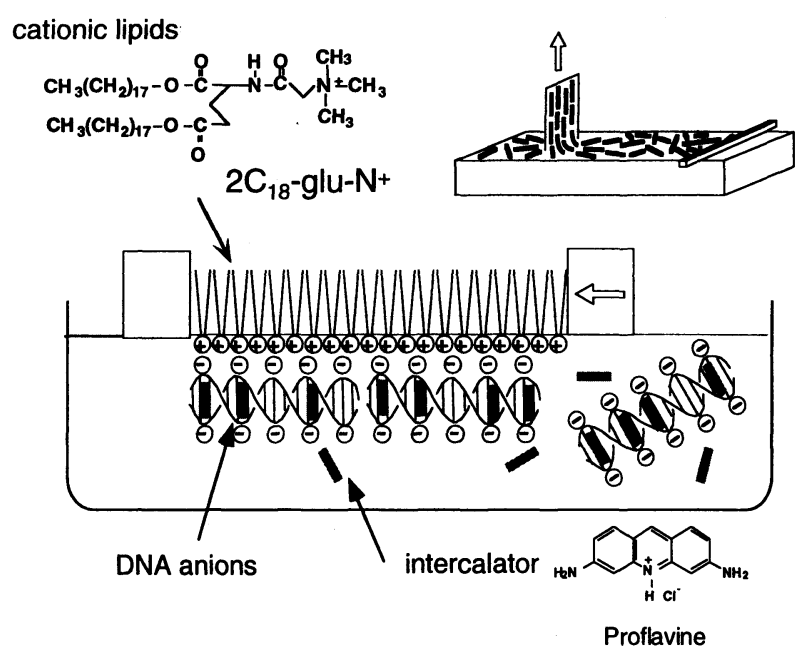

Fig. 4. A schematic illustration of preparation of a DNA-oriented LB film by using polyion complex of DNA/intercalator and cationic lipid monolayers.

could transfer water-soluble DNA strands by complexing with cationic lipid monolayers by vertical dipping LB methods as shown in Fig. 4.

Cationic amphiphiles $2 \mathrm{C}_{18}$-glu- $\mathrm{N}^{+}$were spread on the solution of $10 \mathrm{mM}$ DNA containing $10 \mathrm{mM}$ intercalating dyes (proflavine). The dye-intercalated DNA anions were expected to adsorb to the cationic lipid monolayer due to electrostatic interactions and were transferred on the hydrophobized glass plate at the surface pressure of $35 \mathrm{mN} \mathrm{m}^{-1}$ at $20^{\circ} \mathrm{C}$. From a moving area of a barrier, two layers of the monolayer were confirmed to be transferred in each one cycle (Y-type deposition). When the QCM plate was employed as a transfer plate, the transferred mass can be calculated from frequency decreases (mass increase on the QCM) ${ }^{11), 12}$ It was confirmed that $203 \pm 10 \mathrm{ng}$ of two lipid monolayers and $74 \pm 5$ ng of DNA strands were transferred on the substrate per each dipping cycle, which means $c a .95 \%$ of the monolayer area was covered by DNA molecules.

The LB film of the DNA-/proflavine $/ 2 \mathrm{C}_{18}$-glu- $\mathrm{N}^{+}$ complex was transferred with 44 layers (22 cycles) on one side of a glass plate (total 88 layers on both sides) and measured by polarized absorption spectra in aqueous solution (see Fig. 5). The absorption of the intercalated proflavine at $450 \mathrm{~nm}$ for the light polarized perpendicular to the dipping direction was 2.6 times larger than that for the parallelly polarized light $\left(\mathrm{A}_{\perp} / \mathrm{A}_{/ /}=2.6\right)$. This indicates that absorption moment of the intercalated dyes between base-pairs is aligned perpendicular to the dipping direction. Thus, DNA strands in the LB film are aligned parallel to the dipping direction. 


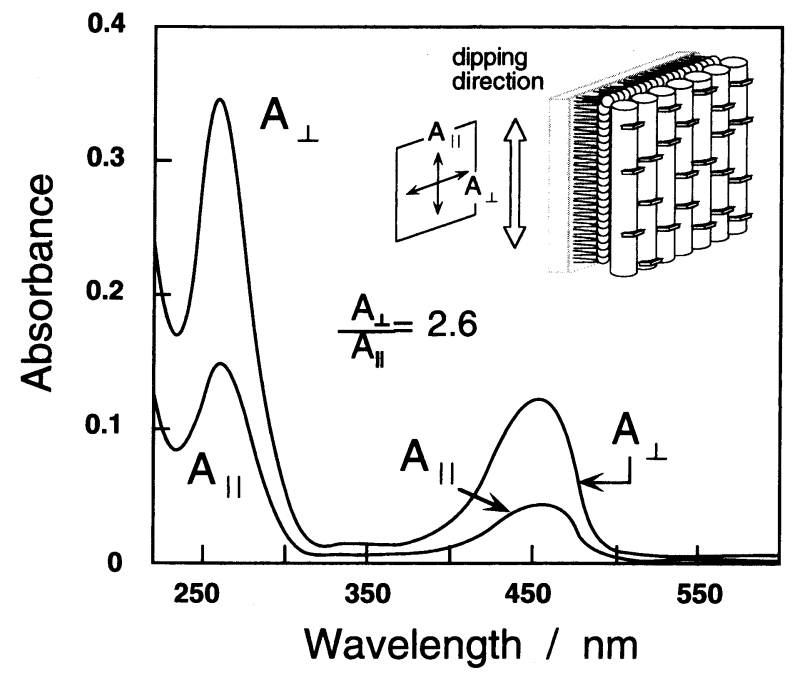

Fig. 5. Polarized absorption spectra of $\mathrm{DNA}^{-} /$proflavine/2C $\mathrm{C}_{18}$-glu$\mathrm{N}^{+}$LB films (88 layers) deposited by vertical dipping method.

When the LB film of the DNA-lipid complex was transferred by a horizontal lifting method on the hydrophobized glass plate, the absorption spectra were independent of the polarized direction of the light (not shown in the figure). This indicates that DNA strands are not aligned in the LB film deposited with a horizontal lifting method. The advantage of the horizontal lifting method is known to deposit the real monolayer structure on the subphase, in contrast to the vertical dipping method. Thus, it is concluded that DNA strands is not oriented on the subphase after and/or during the compression process of the monolayer, but DNA stands are aligned parallel to the dipping direction during vertical dipping process (see Fig. 5).

Anisotropic Conductivity of the DNA Film. Thin films, in which DNA strands were aligned in one direction by stretching the cast film and by vertical LB dipping process, could be expected to show an anisotropic electric conductivity along DNA strands in the film.

The DNA film prepared by cast and stretched method ( $20 \times 10 \mathrm{~mm}$, thickness: $30 \pm 5 \mu \mathrm{m})$ was put on a comb-shaped electrode plate as shown in Fig. 6. The dc conductivity was measured by using an ammeter (R8340A, Advantest, Co., Tokyo, Japan) at $25^{\circ} \mathrm{C}$ in atmosphere or in the vacuumed bottle at $0.1 \mathrm{mmHg}$. When the stretched DNA film was put on the combshaped electrode as DNA strands aligned perpendicularly to two Au electrodes, a large ohmic current $(0-4.1 \mu \mathrm{A})$ was observed that increased linearly with increasing the applied voltage up to $0.1 \mathrm{~V}$. On the contrary, when the film was put as DNA strands aligned parallelly to two

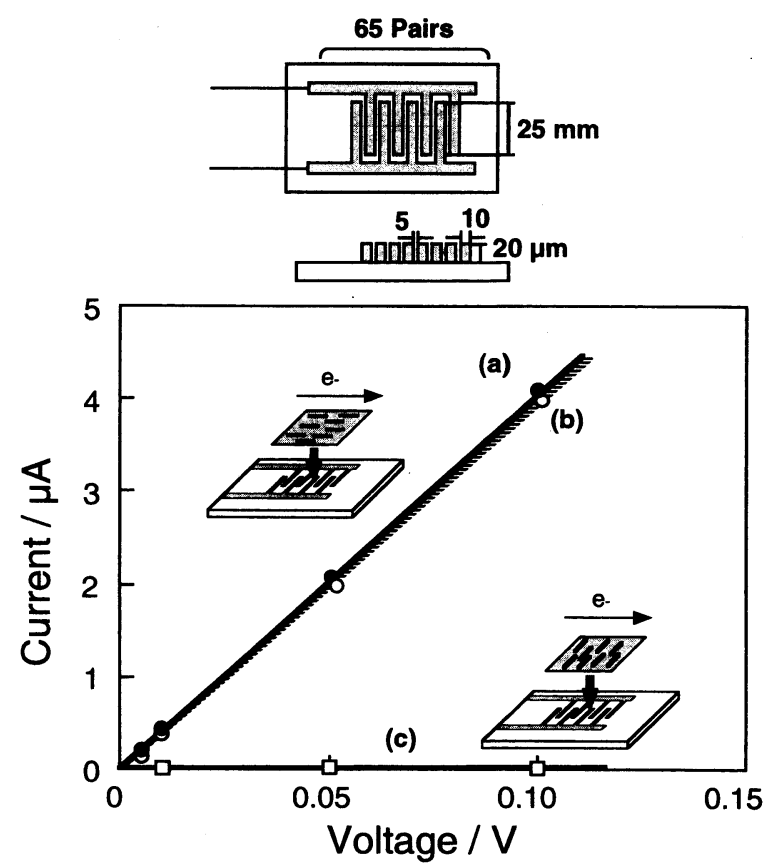

Fig. 6. Electric current of a DNA-aligned cast film $(20 \times 10 \mathrm{~mm}$, thickness: $30 \pm 5 \mu \mathrm{m}$ ) on comb-shaped electrodes at $25^{\circ} \mathrm{C}$. (a) DNA strands in the film was put perpendicularly to two electrodes and measured in atmosphere, (b) the same film of (a) was measured in vacuum at $0.1 \mathrm{mmHg}$, and (c) DNA strands in the film was put parallelly to two electrodes both in vacuum and in atmosphere.

electrodes, electric current was hardly observed even at the voltage of $0.1 \mathrm{v}$ (less than $0.08 \mathrm{nA}$ ). When these experiments were carried out in vacuum at $0.1 \mathrm{mmHg}$ for $24 \mathrm{~h}$, the similar currents were observed. This means that water molecules may bound near phosphate groups in DNA main chains do not play an important role for conductivity.

Conductivity was calculated to be $5.6 \times 10^{-5}$ and $10^{-9}$ $\mathrm{S} \mathrm{cm}{ }^{-1}$ for parallel and perpendicular to DNA strands, respectively. The conductivity of conventional polymers such as polyethylene film was observed to be $c a .10^{-10} \mathrm{~S} \mathrm{~cm}^{-1}$ in this condition. ${ }^{28)}$ The conductivity of $10^{-5} \mathrm{~S} \mathrm{~cm}^{-1}$ in the aligned-DNA flexible film is reasonable as compared with other fragile conventional conductive polymers such as polyacetylene and polyphenylene $\left(10^{-3}\right.$ to $10^{-1} \mathrm{~S} \mathrm{~cm}^{-1}$ without dopants) ${ }^{29)}$

Similar anisotropic conductivity was also observed for the DNA film prepared by LB method, in which 200 layers of the $\mathrm{DNA} / 2 \mathrm{C}_{18}$-glu- $\mathrm{N}^{+}$monolayers were deposited on the same comb-shaped electrode by vertical dipping method (see Fig. 7). When LB films were deposited like DNA strands cross electrodes, the large current was observed, but not DNA strands were 


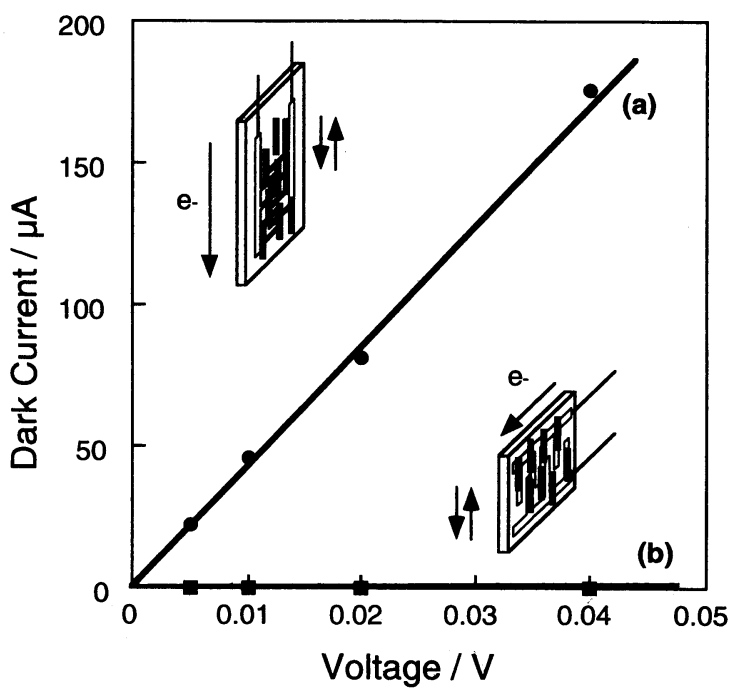

Fig. 7. Electric current of DNA film prepared by LB method (200 layers, thickness: $c a .0 .8 \mu \mathrm{m}$ ) on the same comb-shaped electrodes at $25^{\circ} \mathrm{C}$ as shown in Fig. 6. (a) DNA strands were deposited perpendicularly to two electrodes, and (b) DNA strands were deposited parallelly to two electrodes by the vertical dipping method.

deposited parellelly to the electrodes. However, the electric current along the DNA strands in the LB film was ca. 300 times larger than that in the cast-stretched film. We could not explain precisely the reason showing the larger current in the LB film at present. One of possible reasons seems to be the contact between electrodes and films. DNA strands were directly deposited on Au electrodes in LB method, on the contrary, the DNA-lipid cast film was just pushed on the surface of electrodes.

Conclusion. We have succeeded to produce the DNA-oriented organic thin films by two methods: the uniaxial stretching of DNA-lipid cast film, and the LB vertical transfer of DNA with cationic lipid monolayers. DNA strands can be aligned in one direction by stretching force in the cast film, and by dipping force in the LB film. We have observed the one-dimensional current along DNA strands aligned in films prepared by both caststretched and LB methods.

The DNA-lipid film, in which aligned rod-like strands having hairy alkyl side chains shows thermotropic liquid crystal properties. The aligned-DNA, transparent, self-standing, and flexible film is interesting as a new naturally-occurring functional material, as well as the anisotropic conductive film. For example, the DNA-lipid film is effective as an adsorption filter of carcinogens such as acridine orange and ethidium bromide. The aligned-DNA film also shows polarization of light.

\section{References}

1) Saenger, W. (ed.) (1987) Principles of Nucleic Acid Structure, Springer-Verlag, Berlin.

2) Leslie, A. G. W., Arnott, S., Chandrasekaran, R., and Ratliff, R. L. (1980) J. Mol. Biol. 143, 49-72.

3) Fuller, W. (1967) J. Mol. Biol. 27, 507-524.

4) Alam, T. M., Orban, J., and Drobny, G. (1990) Biochemistry 29, 9610-9617.

5) Alam, T. M., and Drobny, G. P. (1993) Chem. Rev. 91, 15451590.

6) Brandes, R., Vold, R. R., and Kearns, D. R. (1988) Biopolymers 27, 1159-1170.

7) Wada, A. (1964) Biopolymers 2, 361-388.

8) Norden, B., Kubista, M., and Kurucsev, T. (1992) Q. Rev. Biophys 25, 51-170.

9) Strzelecka, T. E., and Rill, R. L. (1987) J. Am. Chem. Soc. 109, 4513-4518.

10) Sauerbrey, G. (1959) Z. Phys. 155, 206-222.

11) Ariga, K., and Okahata, Y. (1989) J. Am. Chem. Soc. 111, 5618-5622.

12) Ariga, K., and Okahata, Y. (1994) Langmuir 10, 3255-3259.

13) Okahata, Y., and Ariga, K. (1987) J. Chem. Soc., Chem. Commun., pp. 1535-1537.

14) Okahata, Y., Ebato, H., and Taguchi, K. (1987) J. Chem. Soc., Chem. Commun., pp. 1363-1365.

15) Thompson, M., Arthur, C. L., and Dhaliwal, G. K. (1986) Anal. Chem. 58, 1206-1209.

16) Evansole, R. C., Miller, J. A., Moran, J. R., and Ward, M. D. (1990) J. Am. Chem. Soc. 112, 3239-3241.

17) Nelson, J. W., and Tinoco, I., Jr. (1984) Biopolymers 23, 213233.

18) Okahata, Y., Ijiro, K., and Matsuzaki, Y. (1993) Langmuir 9, 1921.

19) Stein, R. S. (1958) J. Polym. Sci. 31, 327-334.

20) Skotheim, T. A. (ed.) (1986) Handbook of Conducting Polymers, vols. I and II, Marcel Dekker, New York.

21) Duda, G., and Wegner, G. (1988) Macromol. Chem. Rapid Commun. 9, 495-501.

22) Erbach, R., Hoffmann, B., Schaub, M., and Wegner, G. (1992) Sensors and Actuators, B 6, pp. 211-216.

23) Gaines, G., Jr. (1991) Langmuir 7, 834-839.

24) Karthaus, O., Ringsdorf, H., Tsukruk, V. V., and Wendorff, J. H. (1992) Langmuir 8, 2279-2283.

25) Jones, R., and Tredgold, R. H. (1988) J. Phys. D., Appl. Phys. 21, 449-453.

26) Schwiegk, S., Vahlenkamp, T., Xu, Y., and Wegner, G. (1992) Macromolecules 25, 2513-2525.

27) Sukhorukhov, G. B., Yerokhin, V. V., and Yu, A. (1992) Biophysics 38, 243-248.

28) Skotheim, T. A. (1986) Handbook of Conductive Polymers, Marcel Dekker, New York.

29) Yamamoto, T., Maruyama, T., Zhou, Z., Ito, T., Fukuda, T., Yoneda, Y., Begum, F., Ikeda, T., Sasaki, S., Takezoe, H., Fukuda, A., and Kubota, K. (1994) J. Am. Chem. Soc. 116, 4832-4845. 